\title{
Alexandra MICHALEWSKI, La Puissance de l'intelligible. La théorie plotinienne des Formes au miroir de l'héritage médioplatonicien
}

\section{Sylvain Roux}

\section{(2) OpenEdition}

\section{Journals}

Édition électronique

URL : https://journals.openedition.org/philosant/479

DOI : 10.4000/philosant.479

ISSN : 2648-2789

Éditeur

Éditions Vrin

\section{Édition imprimée}

Date de publication : 24 novembre 2015

Pagination : 288-291

ISBN : 978-2-7574-1141-4

ISSN : 1634-4561

\section{Référence électronique}

Sylvain Roux, «Alexandra michalewskı, La Puissance de l'intelligible. La théorie plotinienne des Formes au miroir de l'héritage médioplatonicien », Philosophie antique [En ligne], 15 | 2015, mis en ligne le 01

novembre 2018, consulté le 03 décembre 2022. URL : http://journals.openedition.org/philosant/479 ; DOI : https://doi.org/10.4000/philosant.479

\section{(c)}

Creative Commons - Attribution - Pas d'Utilisation Commerciale - Pas de Modification 4.0 International - CC BY-NC-ND 4.0

https://creativecommons.org/licenses/by-nc-nd/4.0/ 
Alexandra MICHALEWSKI, La Puissance de Pintelligible. La théorie plotinienne des Formes au miroir de Phéritage médioplatonicien, Leuven, Leuven University Press, 2014 (Ancient and Medieval Philosophy, Series 1), 280 p. ISBN 9789462700024

L'ouvrage que présente Alexandra Michalewski reprend et articule trois questions classiques dans les études plotiniennes. La première est celle des origines et des sources de la doctrine des Formes intelligibles dans la pensée de Plotin. Si cette doctrine puise dans la pensée platonicienne ses éléments principaux, elle ne peut pourtant s'expliquer entièrement par elle. Cette dernière a connu, en effet, de multiples remaniements et adaptations, elle a suscité de multiples débats, de sorte qu'elle s'est transmise considérablement modifiée à un auteur comme Plotin. C'est pourquoi il apparaît essentiel, pour mesurer l'originalité de la pensée de ce dernier, de saisir les emprunts autant que les déplacements qu'elle a pu opérer par rapport à la tradition platonicienne immédiate, représentée par ce que l'historiographie contemporaine a appelé le «médioplatonisme ». La seconde question concerne le contenu doctrinal de la théorie des Formes. Les travaux qui lui ont été consacrés ont eu pour objectif d'en présenter les aspects principaux et d'en proposer à la fois une reconstitution et une synthèse. La dernière question concerne un point spécifique de cette théorie, sur lequel le titre de l'ouvrage d'A. Michalewski attire immédiatement l'attention. Il s'agit du problème de la causalité des Formes intelligibles, ou, selon une terminologie moins aristotélicienne et plus proprement plotinienne, de leur puissance. Cette question n'est évidemment pas nouvelle. Aristote s'interrogeait déjà sur le fait de savoir en quel sens les Formes peuvent, selon Platon, jouer le rôle de cause (par exemple en Métaphysique, A 9) et l'on sait qu'il se montrait sévère à cet égard puisqu'il estimait qu'elles ne peuvent être considérées ni comme des causes motrices (principe de mouvement et de changement), ni comme des causes exemplaires (paradigmes).

C'est cet ensemble de questions que reprend l'ouvrage d'A. Michalewski. Le premier point est longuement abordé par l'ouvrage, puisque celui-ci lui consacre la première des deux parties qu'il comporte. L'auteur insiste particulièrement sur deux aspects qui caractérisent le médioplatonisme. D’une part, les Formes platoniciennes apparaissent, dans le contexte historique et philosophique du médioplatonisme, comme les «pensées du dieu » c'est-à-dire du démiurge du monde sensible, selon les indications fournies par le Timée. D’autre part, le médioplatonisme adopte sur un autre point une lecture originale de ce dialogue platonicien. Les interprétations antérieures considéraient que la physique et plus largement la cosmologie platoniciennes pouvaient se réduire à une théorie comprenant deux principes principaux, la matière et le dieu, laissant de côté le rôle et le statut des Formes intelligibles dans ce contexte physique et cosmologique. Au contraire, le médioplatonisme propose une lecture «tri-principielle du Timée (la matière, le dieu, les Formes). Or, cela conduit à donner aux Formes une importance nouvelle, puisqu'elles constituent, dès lors, un principe à part entière, et à 
leur reconnaître une véritable puissance causale. Plotin est l'héritier de cette nouvelle conception mais il s'en démarque aussi sur plusieurs points. S'il reconnaît l'existence et l'importance de la puissance causale des Formes, il ne reprend pas la conception médioplatonicienne qui comprend cette puissance à la lumière d'un modèle qu'A. Michalewski désigne par l'expression d'«artificialisme démiurgique ». Selon ce modèle, le dieu est la véritable puissance causale et il œuvre en vue d'une fin en se servant des Formes intelligibles comme moyens et comme instruments. Le problème rencontré par Plotin peut dès lors être présenté de manière très précise : d'un côté, il lui faut échapper à la conception stoïcienne, qui s'apparente, selon l'auteur, à une forme de «vitalisme immanent», puisque le dieu stoïcien œuvre dans le monde en y étant lui-même présent, de l'autre il lui faut échapper à la conception médioplatonicienne qui, pour lutter contre la conception précédente, opte pour une forme d' « artificialisme démiurgique » dans laquelle la transcendance du divin se trouve réaffirmée mais dans le cadre d'une conception du dieu-artisan. Contre ces deux tendances, Plotin cherche à ouvrir une nouvelle voie que l'auteur appelle « vitalisme intelligible» (p. 3), et qui, à la fois, maintient la transcendance du dieu et de l'intelligible et accorde aux Formes une puissance causale dégagée pourtant de toute forme d'artificialisme.

La seconde partie de l'ouvrage présente la théorie plotinienne des Formes mais surtout la conception de la puissance que Plotin leur prête. Concernant le premier point, l'auteur aborde certains des problèmes les plus importants de cette théorie. Tout d'abord, Plotin fait dépendre les Formes intelligibles d'un premier principe qui les fait apparaître tout en étant radicalement différent d'elles (c'est la thèse célèbre, exposée par exemple dans le traité 38 (VI 7), 17, selon laquelle le Bien donne ce qu'il n'a pas). Ce paradoxe suscite les plus grandes difficultés, qui sont bien perceptibles à travers le recours que fait Plotin à la théorie dite des deux actes. Celle-ci permet de dire la transcendance du premier principe selon le modèle d'un acte substantiel qui produit un acte second mais elle instaure, entre le terme engendré et ce principe, une ressemblance qui est celle d'une image par rapport à son modèle, et qui peut affaiblir cette transcendance elle-même. La théorie plotinienne des Formes insiste par ailleurs sur l'identité de l'Intellect et des Formes intelligibles. Cette thèse signifie que les Formes ne sont pas extérieures à l'Intellect mais aussi qu'elles ne sont pas extérieures les unes aux autres puisque chacune enveloppe et exprime toutes les autres. Mais, et c'est là encore un des paradoxes de la pensée de Plotin, l'Intellect ne se confond pourtant pas avec les Formes : celles-ci existent de manière distincte (distinction qui est noétique et non point spatiale), tout en ne formant avec l'Intellect qu'une seule et même réalité. Enfin, Plotin soutient une thèse qui a considérablement attiré l'attention des commentateurs par son étrangeté et sa nouveauté, celle de l'existence de Formes d'individus. Mais l'interprétation de cette théorie est particulièrement délicate et divise les commentateurs. Comme nous le verrons plus loin, l'auteur en présente une lecture originale. 
Le second point abordé par la seconde partie est celui de la puissance causale des Formes. Il s'agit ici d'expliquer comment les Formes peuvent posséder en ellesmêmes et par elles-mêmes une telle puissance, que ne leur reconnaissait pas la conception médioplatonicienne. La réponse à cette question nous amène au cœur de l'ouvrage. Selon A. Michalewski, c'est parce que Plotin accorde une autarcie aux Formes intelligibles qu'il peut en même temps leur reconnaître une véritable puissance causale. L'autarcie explique en effet que les Formes puissent être considérées comme des principes à part entière. Ici, l'auteur peut s'appuyer sur le traité 38 (VI 7), 2, qui montre que les Formes possèdent en elles-mêmes (c'est-à-dire dans l'Intellect) leur propre pourquoi et leur propre raison d'être, mais aussi sur le traité 49 (V 3), 17, qui attribue spécifiquement l'autarcie à l'Intellect puisque l'Un se situe au-delà de tout, y compris de toute forme d'autarcie. Mais ce dernier texte souligne aussi que l'Un donne cette autarcie à l'Intellect. Le paradoxe est donc le suivant : les Formes dépendent bien du premier principe mais sont pourtant autosuffisantes et auto-constituantes. En effet, c'est en se retournant vers l'Un que ce qui en émane se trouve déterminé et se constitue comme Intellect. L'Intellect est autarcique, il se donne à lui-même sa propre détermination tout en étant subordonné par cela même au Bien. Là est la source de toute productivité dans le système plotinien : la contemplation du principe supérieur conduit à l'apparition de réalités nouvelles et c'est ainsi que les Formes intelligibles peuvent produire en échappant à toute forme d'artificialisme et de représentation. Même si Plotin reprend le terme « démiurge » pour l'appliquer à l'Intellect, ce n'est donc jamais pour reproduire un schéma artificialiste, c'est-à-dire pour faire de l'Intellect un artisan, mais pour repenser la causalité de façon dynamique comme une puissance propre aux êtres susceptibles de contemplation et qui produisent sans se représenter de fins.

Le mérite de cet ouvrage est donc de fournir des éléments pour résoudre certains des paradoxes de la pensée de Plotin. Ainsi, concernant la puissance du premier principe, A. Michalewski montre que si l'Un donne ce qu'il n'a pas, il n'en donne pas moins quelque chose de lui-même. Cependant, ce produit de l'Un est d'abord indéterminé et ce n'est que par le retour vers son principe qu'il prend sa forme d'être intelligible. Ce que ne donne pas l'Un, c'est l'être déterminé car la détermination est, en quelque sorte, le fait même de l'engendré et ce que l'Un donne n'est qu'une puissance de se constituer soi-même. De même, la théorie si complexe de l'existence des Formes d'individus peut trouver une explication si l'on rappelle qu'il n'y a pas au sens strict de Formes d'individus. Des Formes, il faut distinguer les intellects des âmes individuelles, qui restent toujours auprès de l'intelligible. Or, les âmes individuelles possèdent les logoi des différents êtres qu'elles animeront au cours d'un cycle d'incarnations et, par là même, elles contiennent ce qui sera développé par différents individus au cours de ces incarnations. De la sorte, il existe un principe intelligible de l'individualité mais qui n'est pas une forme intelligible au sens strict puisqu'il s'agit des logoi qui spécifient 
les individus. On voit donc que la pensée de Plotin affectionne les paradoxes. L'ouvrage d'A. Michalewski en présente et en affronte quelques-uns : l'Un peut donner parce qu'il ne possède pas ce qu'il donne, les Formes sont autarciques parce qu'elles dépendent du premier principe, l'individualité trouve son principe dans l'intelligible. Mais elle montre qu'ils peuvent se résoudre si l'on reconstitue la théorie originale de la production et de la puissance causale à l'intérieur de laquelle ils prennent place : la puissance des Formes intelligibles s'explique alors par un dynamisme qui leur est propre et dont elles héritent pourtant de leur terme supérieur. Ce qui peut apparaître contradictoire dans la pensée de Plotin est justement ce qui permet de penser la puissance causale de l'Intellect et finalement d'autres réalités à l'exclusion de la matière. Cette intuition, qui était déjà celle de Jean Trouillard dans la Procession plotinienne, trouve dans l'ouvrage d'A. Michalewski une nouvelle confirmation.

Sylvain Roux

Université de Poitiers

Philippe Soulier, Simplicius et Pinfini, Paris, Les Belles Lettres, 2014, « Anagôgê », 595 p. ISBN 978-2-251-42016-5

L'ouvrage, précédé d'une préface de $\mathrm{Ph}$. Hoffmann, reprend la première partie de la thèse que $\mathrm{Ph}$. Soulier a soutenue en 2010 . Il présente une étude très précise des enjeux philosophiques des pages que Simplicius consacre au commentaire des chapitres 4 à 8 du livre III de la Physique d'Aristote (In Physicam, 451-517 Diels). Il contient également une annexe présentant un résumé analytique du texte de Simplicius, une bibliographie, trois index (noms anciens, auteurs modernes, passages cités) et une table des matières très détaillée.

Dans la première partie («La thèse philosophique de Simplicius sur l'æ̋ $\pi \varepsilon l-$ pov », p. 25-73), après avoir présenté brièvement deux interprétations modernes de l'infini aristotélicien (Hintikka et Wieland), l'auteur en vient à celle de Simplicius, qu'il qualifie de « réaliste ». Il soutient que, en réduisant le concept aristotélicien de « pensée » (vónoı, Physique, III 4 et 8 ) à une « représentation » $(\dot{\varepsilon} \pi i v \circ \alpha)$ ou « imagination » $(\phi \alpha \nu \tau \alpha \sigma i \alpha)$, Simplicius procède à une « démystification conceptuelle » qui permet de mieux mettre en évidence la « réalité du procès à l'infini ». Soulier voit dans l'interprétation de la vónбıs en termes de $\phi \alpha \nu$ $\tau \alpha \sigma i \alpha$ ou d'ż $\pi$ ivoı $\alpha$ un geste fort ; cependant on pourrait se demander si Simplicius ne se contente pas d'expliciter la pensée d'Aristote (l'exemple d'Aristote consistant à penser un homme faisant plusieurs fois sa taille ou excédant la taille de la ville, Phys. III 8, 208a16 sqq.). Par ailleurs, il semble y avoir une équivoque dans la façon dont l'abstraction est convoquée. Si tout processus d'abstraction relève de la pensée ou de la représentation, la réciproque est-elle vraie ? Quand Aristote parle de vónбis en Physique III, il a bien en tête une pensée contrefactuelle (d'où l'interprétation de Simplicius en termes de $\phi \alpha \nu \tau \alpha \sigma i \alpha$, qui semble assez vraisemblable), une « représentation fictive » (p. 57), un « produit spéculatif d'une imagination 